\title{
Design and fabrication of a $\mathrm{SiO}_{x} / \mathrm{ITO}$ double-layer anti-reflective coating for heterojunction silicon solar cells
}

\author{
D. Zhang ${ }^{\text {a,* }}$, I.A. Digdaya ${ }^{\text {a }}$, R. Santbergen ${ }^{\text {a }}$, R.A.C.M.M. van Swaaij ${ }^{\text {a }}$, P. Bronsveld ${ }^{\mathrm{b}}$, \\ M. Zeman ${ }^{\text {a }}$, J.A.M. van Roosmalen ${ }^{\text {b }}$, A.W. Weeber ${ }^{\text {b }}$ \\ a Photovoltaic Materials and Devices, Delft University of Technology, P.O. Box 5031, 2600 GA Delft, The Netherlands \\ ${ }^{\mathrm{b}}$ ECN Solar Energy, P.O. Box 1, 1755 ZG Petten, The Netherlands
}

\section{A R T I C L E I N F O}

\section{Article history:}

Received 24 January 2013

Received in revised form

15 May 2013

Accepted 21 May 2013

\section{Keywords:}

Heterojunction silicon solar cell

Anti-reflective coating

\begin{abstract}
A B S T R A C T
In this contribution optical simulations of both flat and textured heterojunction silicon solar cells are presented and verified experimentally. Using Advanced Semiconductor Analysis (ASA) software, we optimize a double-layer anti-reflective (AR) coating, which has an additional $\mathrm{SiO}_{x}$ film on the top of the existing indium tin oxide (ITO) coating. Our approach is based on maximizing the absorbance of the crystalline silicon (c-Si) wafer, which is strongly correlated with the solar cell's short circuit current $\left(J_{\text {sc }}\right.$ ). Our simulations show that for a flat heterojunction silicon solar cell c-Si absorbance can increase by using a double-layer AR coating instead of a single-layer AR coating. As predicted by the simulations, experimental devices show corresponding $J_{\mathrm{sc}}$ increase, leading to the increase of the solar cell efficiency. On a textured heterojunction silicon solar cell the incident light travels an oblique path through the AR coating and we use an advanced ray-tracing model to optimize the single and double-layer AR coating for this case. Our simulations show that for the textured heterojunction silicon solar cell, reflection losses are lower but parasitic absorption losses in the ITO and amorphous silicon layers play a more important role. Using a double-layer AR coating not only reduces reflection losses further, but because a thinner ITO layer can be used it also reduces parasitic absorption losses. Experimentally, our textured heterojunction silicon solar cell with a double-layer AR coating shows that the $J_{\mathrm{sc}}$ (active area) of $40.5 \mathrm{~mA} / \mathrm{cm}^{2}$ and an efficiency of $19.0 \%$.
\end{abstract}

(c) 2013 Elsevier B.V. All rights reserved.

\section{Introduction}

The heterojunction silicon solar cell is attracting more and more attention in both photovoltaic research and industry. The reason is that the heterojunction silicon solar cell has high efficiency [1,2], lower thermal budget, shorter processing time, and potentially lower processing cost compared to the conventional diffused homojunction crystalline silicon (c-Si) solar cell [3]. As is the case for homojunction silicon solar cells, there are also optical losses such as reflection losses and metallic shading losses in heterojunction cells. The high refractive index of the hydrogenated amorphous silicon (a-Si:H) emitter in the heterojunction cell and the c-Si emitter in the homojunction cell results in high reflection losses ( $>40 \%$ in the wavelength range from 300 to $1200 \mathrm{~nm}$ ). Therefore, anti-reflection measures are very important

\footnotetext{
* Corresponding author. Tel.: +31 628637175.

E-mail addresses: dongzhangdz@gmail.com (D. Zhang), ibadillah. digdaya@gmail.com (I.A. Digdaya), r.santbergen@tudelft.nl

(R. Santbergen), r.a.c.m.m.vanSwaaij@tudelft.nl (R.A.C.M.M. van Swaaij), bronsveld@ecn.nl (P. Bronsveld), m.zeman@tudelft.nl (M. Zeman), vanroosmalen@ecn.nl (J.A.M. van Roosmalen), weeber@ecn.nl (A.W. Weeber).
}

for reducing reflection losses and increasing the light absorption in c-Si. In the case of homojunction silicon solar cells, silicon nitride is normally used as anti-reflective (AR) coating. For heterojunction silicon solar cells, the transparent indium tin oxide (ITO) front contact layer is also designed for this AR purpose.

For the heterojunction silicon solar cell, ITO can absorb light in the ultraviolet regime due to its large band gap as well as in the infrared regime due to its free carrier absorption. In addition the high absorption coefficient of a-Si:H leads to high absorption in these thin layers at wavelengths shorter than $700 \mathrm{~nm}$, further contributing to the parasitic absorption losses [4]. These absorption losses can reduce the benefits gained from the AR coating, making the design of an AR coating more complicated for a c-Si homojunction cell. In 2012, Holman et al. showed that how ITO, and p-type and intrinsic a-Si:H layers in the front side of the heterojunction silicon solar cell contribute to the current losses by comparing EQE and simulated absorption in c-Si of different solarcell structures at wavelengths from 300 to $600 \mathrm{~nm}$ [5]. They concluded that no light was absorbed in ITO and p-type a-Si:H contributes to the photocurrent, whereas $20-50 \%$ of the light absorbed in intrinsic a-Si:H can contribute to this current. In this contribution, we present an accurate optical analysis of heterojunction 
silicon solar cells in the wavelength range from $300 \mathrm{~nm}$ to $1200 \mathrm{~nm}$. Based on our optical analysis, we show that for AR coating design the short-circuit current density $\left(J_{s c}\right)$ can be maximized by optical simulations alone, provided that the c-Si wafer absorbance is maximized since this absorbance dominates the $J_{\text {sc. }}$. We show that this procedure is more accurate than simply minimizing the reflectance due to the influence of parasitic absorption of front layers. In this contribution, we illustrate this approach for designing a double-layer AR coating of both a flat and textured heterojunction silicon solar cell. In order to validate our model, solar cells with the optimized AR coating are fabricated.

\section{Experimental}

The structure and layer thicknesses of the heterojunction silicon solar cells with single and double layer AR coating that we process in our facility are shown in Fig. 1. The same structure is
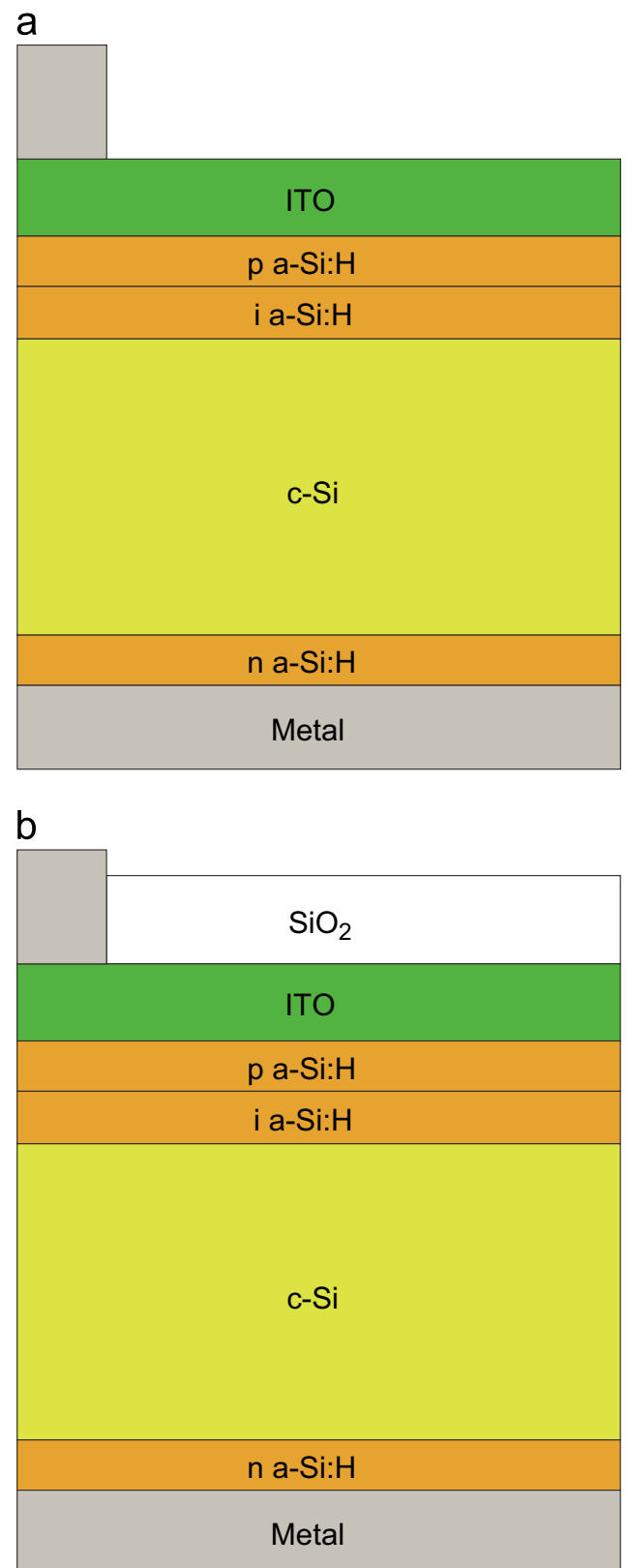

Fig. 1. The structure of the heterojunction silicon solar cell (a) with a single-layer AR coating of ITO and (b) with a double-layer AR coating of $\mathrm{SiO}_{x} / \mathrm{ITO}$. also used for simulations using ASA computer software [6,7]. The area of the solar cells is $0.16 \mathrm{~cm}^{2}$. For the solar cells, n-type c-Si (111) FZ wafers are used with a thickness of about $280 \mu \mathrm{m}$ and selected from a batch with resistivity between 2 and $5 \Omega \mathrm{cm}$. On the back side of the c-Si wafer, a 9 nm thick n-type a-Si:H layer and metal are deposited. On the front side, $5 \mathrm{~nm}$ thick intrinsic and $5 \mathrm{~nm}$ thick p-type a-Si:H layers are deposited, followed by an ITO layer and metal grid. For a-Si:H deposition, radio frequency (rf) plasma enhanced chemical vapor deposition (PECVD) is used, whereas ITO is deposited by rf sputtering. Our ITO has a mobility of $42 \mathrm{~cm}^{2} / \mathrm{V} \mathrm{s}$ and the carrier density is $7 \times 10^{20} \mathrm{~cm}^{-3}$. The metal front and back contacts are deposited by thermal and electronbeam evaporation, and $\mathrm{SiO}_{x}$ by electron-beam evaporation.

In order to execute an accurate optical simulation, the complex refractive indices of all materials used in the heterojunction silicon solar cell are characterized. Transmittance and reflectance of the ITO layer and c-Si are measured by a Perkin-Elmer Lambda 950 UV-vis spectrometer and fitted by SCOUT software to obtain their complex refractive indices [8]. Complex refractive indices of $\mathrm{SiO}_{x}$, p-type, n-type, and intrinsic a-Si:H are obtained from Woollam spectroscopic ellipsometry measurements of individual layers deposited on c-Si wafers. The complex refractive indices of the metal back contact are taken from literature [9]. Thin-film optics is used for the optical simulation on the flat solar cell, taking into account the interference between multiple reflections [10]. The optical effects of the textured surface are described by an advanced ray-tracing model since the feature size is much larger than the wavelengths we investigate [11-13]. This model can also treat the optical interference of thin films on textured interfaces [14]. The reflectance of the solar cells with the structures shown in Fig. 1 is also measured by a Perkin-Elmer Lambda 950 UV-vis spectrometer and the external quantum efficiency (EQE) is measured by a setup developed in-house. These optical measurements are carried out on the active area, i.e. parts of the solar cell that are not covered by the metal grid. $J-V$ characterization is carried out using an Oriel Corporation solar simulator. To avoid errors due to spectral mismatch of the solar simulator, the $J_{\mathrm{sc}}$ is calibrated by the $J_{\text {sc }}$ calculated from EQE measurements. The $J_{\mathrm{sc}}$ and calculated efficiency values presented in this article refer to the active area of the solar cell. Therefore, the shadow losses designed to be $10 \%$ without any optimization are not taken into consideration.

\section{Results and discussion}

In order to compare the effectiveness of an AR coating on a solar cell, it is important to take the AM 1.5 solar spectrum into account. For this investigation we have chosen the wavelength range from $300 \mathrm{~nm}$ to $1200 \mathrm{~nm}$ of the solar spectrum. The reason is that for wavelengths shorter than $300 \mathrm{~nm}$, the spectral power density in the AM 1.5 spectrum is almost zero, while photons with wavelengths longer than $1200 \mathrm{~nm}$ are hardly absorbed by the c-Si. In this work, we define the weighted average reflectance $(\bar{R})$ of the solar cell to be the fraction of reflected photons in the AM 1.5 solar spectrum:

$$
\bar{R}(\%)=\frac{\int_{300}^{1200} R(\lambda) \Phi(\lambda) d \lambda}{\int_{300}^{1200} \Phi(\lambda) d \lambda} \times 100 \%
$$

Here $R(\lambda)$ is the reflectance of the solar cell shown in Fig. 1 and $\Phi$ $(\lambda)$ is the photon flux density of the AM 1.5 spectrum, both as a function of wavelength $\lambda$. In Fig. 2, the numerator and denominator of Eq. (1) are shown as black and blue lines, respectively. The $\bar{R}$ is usually treated as a cost function for reflection minimization.

Similarly, the weighted average absorbance $(\bar{A})$ in each layer of the solar cell is defined to be the fraction of absorbed photons in 


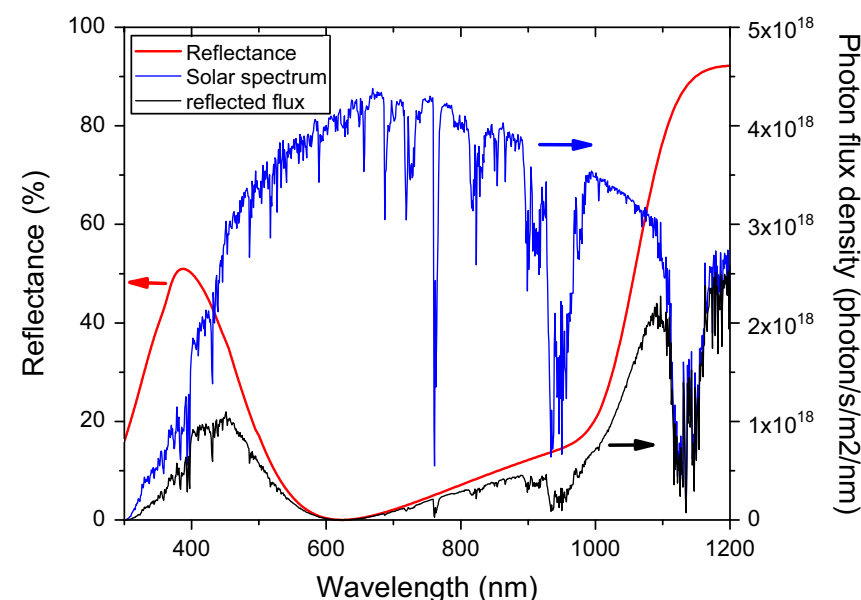

Fig. 2. Measured reflectance of the heterojunction silicon solar cell (red), photon flux density in the solar spectrum of AM 1.5 (blue) and reflected photon flux density on the solar cell (black) as a function of wavelength. (For interpretation of the references to color in this figure legend, the reader is referred to the web version of this article.)

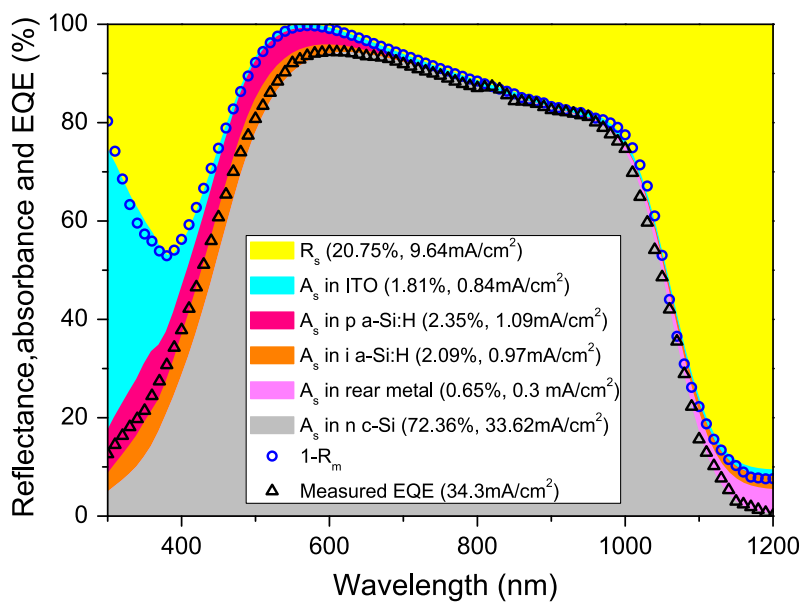

Fig. 3. Optical simulation of a flat heterojunction silicon solar cell and its reflectance and EQE measurement. $\bar{R}$ and $\bar{A}$ as well as their contributing current densities are calculated.

the AM 1.5 solar spectrum:

$\bar{A}(\%)=\frac{\int_{300}^{1200} A(\lambda) \Phi(\lambda) d \lambda}{\int_{300}^{1200} \Phi(\lambda) d \lambda} \times 100 \%$

Here $A(\lambda)$ is the absorbance of one layer in the solar cell. Assuming that all photons with wavelengths ranging from $300 \mathrm{~nm}$ to $1200 \mathrm{~nm}$ of the AM 1.5 solar spectrum can be absorbed by the solar cell, the maximum current density is $46.46 \mathrm{~mA} / \mathrm{cm}^{2}$ when there are no optical and electrical losses. Therefore, for instance, the maximum current density contributed by c-Si $\left(\mathrm{J}_{\mathrm{c}-\mathrm{Si}}\right)$ can be calculated as

$J_{c-S i}\left(\mathrm{~mA} / \mathrm{cm}^{2}\right)=46.46 \times \bar{A}_{c-S i}$

where $\bar{A}_{c-S i}$ is weighted average absorbance in c-Si. This calculation can also be applied to other layers of the solar cell.

With ASA software, the reflectance of the solar cell and absorbance in each layer can be simulated, using the complex refractive indices of each layer and layer thicknesses as input. Fig. 3 shows the optical simulation results of a solar cell with a structure as in Fig. 1(a) in which ITO is $71 \mathrm{~nm}$ thick. The corresponding reflectance and EQE measurements are shown in the same figure for comparison. The measured fraction of the light entering the solar cell, which is unity minus reflectance, has an excellent agreement with the simulation, indicating the validity of the optical model in ASA. The measured EQE curve closely follows the simulated absorbance in c-Si in Fig. 3. Especially in the wavelength range of 600-1200 $\mathrm{nm}$ there is a close match between $\mathrm{EQE}$ and the absorbance in $\mathrm{c}-\mathrm{Si}$, implying that the recombination loss is negligible for this cell. Below $600 \mathrm{~nm}$ there are minor deviations between EQE and the absorbance in c-Si. We attribute this to a wavelength-dependent contribution of light absorption in the intrinsic a-Si:H to the EQE. The same phenomenon is also reported by Holman et al. [5]. A new observation however, is that in the wavelength range of $300 \mathrm{~nm}-400 \mathrm{~nm}$ a very small fraction of the light absorbed in p-type a-Si:H also contributes to the current output. Nevertheless, the light absorption in c-Si contributes $33.6 \mathrm{~mA} / \mathrm{cm}^{2}$ out of the total $J_{\mathrm{sc}}$ of $34.3 \mathrm{~mA} / \mathrm{cm}^{2}$, and there is only $0.7 \mathrm{~mA} / \mathrm{cm}^{2}$ from the absorbance of a-Si:H. Note that the more conventional approach of minimizing reflectance implies maximizing the total absorbance, including parasitic absorption losses in ITO, p-type a-Si:H and rear metal. In the next section we will illustrate that different design approaches can lead to different results and we argue that it is more accurate to design an AR coating for heterojunction silicon solar cells by considering the absorbance in c-Si.

\subsection{Optical simulation of the heterojunction silicon solar cell on a flat surface}

Using ASA we calculate the $\bar{R}$ and $\bar{A}_{c-S i}$ of heterojunction silicon solar cells (Fig. 1(a)) with different ITO thicknesses as demonstrated in Fig. 4. According to the simulation, the $\bar{A}_{c-S i}$ shows almost exactly the opposite trend as that of the $\bar{R}$. Therefore, both the approach of maximizing $\bar{A}_{c-S i}$ and the approach of minimizing $\bar{R}$ give the same optimum ITO thickness of $80 \mathrm{~nm}$.

From ellipsometry measurements we have found that our $\mathrm{SiO}_{x}$ has a refractive index of about 1.5. Because this refractive index is in between the refractive indices of air and ITO we can apply $\mathrm{SiO}_{x}$ on the top of ITO to form a double-layer AR coating. Similarly, we can calculate the change of $\bar{R}$ and $\bar{A}_{c-\mathrm{Si}}$ when the $\mathrm{SiO}_{x}$ thickness and the ITO thickness vary independently. Fig. 5(a) and (b) shows the $\bar{R}$ and $\bar{A}_{c-\mathrm{Si}}$ as a function of ITO and $\mathrm{SiO}_{x}$ thicknesses, respectively. The patterns in Fig. 5(a) and (b) are similar. The optimum thicknesses for $\mathrm{SiO}_{x}$ and ITO to obtain the minimum $\bar{R}$ of the solar cell are respectively $70 \mathrm{~nm}$ and $60 \mathrm{~nm}$. The same thickness combination gives the maximum $\bar{A}_{c-\mathrm{Si}}$. Therefore, also in case of the double-layer AR coating, minimizing reflectance and maximizing transmittance give the same result in flat heterojunction silicon solar cells. Note that the optimum thickness actually

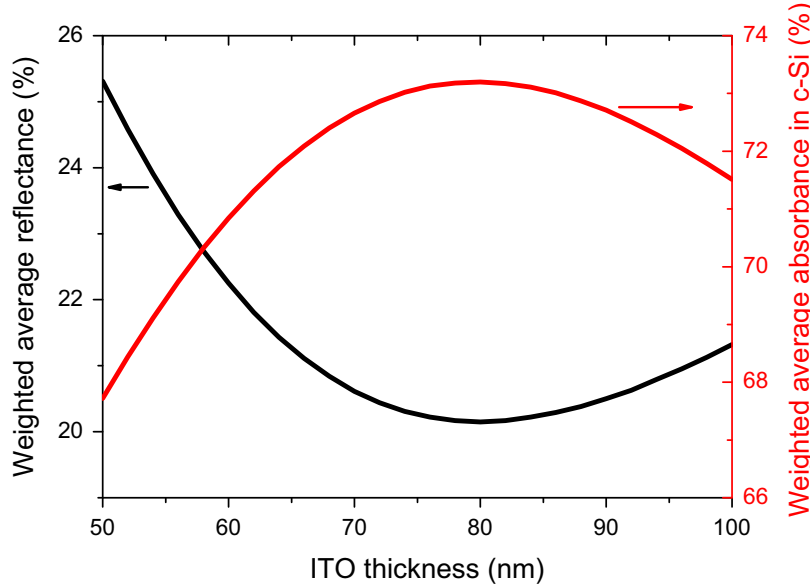

Fig. 4. The simulated $\bar{R}$ and $\bar{A}_{c-S \mathrm{i}}$ as a function of ITO thicknesses. 
a
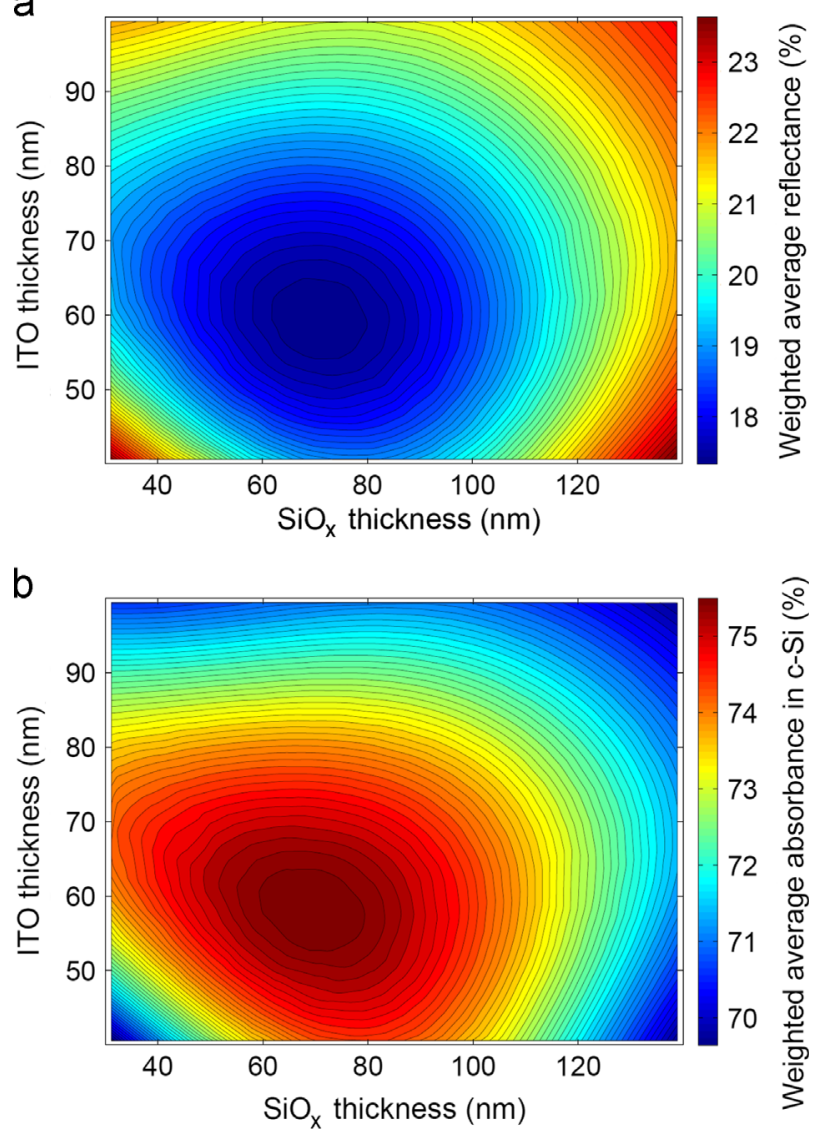

Fig. 5. The simulated (a) $\bar{R}$ and (b) $\bar{A}_{c-\mathrm{Si}}$ as a function of ITO and $\mathrm{SiO}_{x}$ thicknesses.

has a quite high tolerance, meaning that a deviation of $5 \mathrm{~nm}$ from the optimum value gives a reflectance or absorbance difference of less than $0.1 \%$ (absolute value) and for a deviation of even $10 \mathrm{~nm}$ it is less than $1 \%$. From a practical point of view this is desirable, since no high accuracy of thickness is required.

It is interesting to look in more detail at the optical benefits we gain from the double layer AR coating compared to the singlelayer AR coating, which can be seen by comparing Figs. 4 and 5 . The simulations suggest that by applying the double-layer AR coating, the minimum $\bar{R}$ is reduced from $20.2 \%$ to $17.3 \%$, implying a potential current density gain of $1.35 \mathrm{~mA} / \mathrm{cm}^{2}$ from the reduction of reflection losses. However, the maximum $\bar{A}_{c-S i}$ is increased from $73.2 \%$ to $75.6 \%$, indicating a more realistic current density gain of $1.12 \mathrm{~mA} / \mathrm{cm}^{2}$. The difference can be explained by the influence of parasitic absorption in ITO and a-Si:H.

\subsection{Optical measurement of the heterojunction silicon solar cell on a flat surface}

In order to verify the accuracy of our simulation, heterojunction silicon solar cells with ITO thicknesses of 53, 71 and $94 \mathrm{~nm}$ have been made and the reflectance of these cells is measured and then compared to ASA simulations. In the simulation, the solar-cell structure is defined as depicted in Fig. 1 and the complex refractive indices of each layer, which are obtained from the optical characterization experiments described in Section 2, are used. In order to match the simulation results to the experimental data for each solar cell, only the thicknesses of the ITO and $\mathrm{SiO}_{x}$ layers are varied slightly in the simulations to account for layer thickness nonuniformity in the experimental solar cell, while all other thicknesses are as indicated in Fig. 1. The results are shown in Fig. 6(a). a

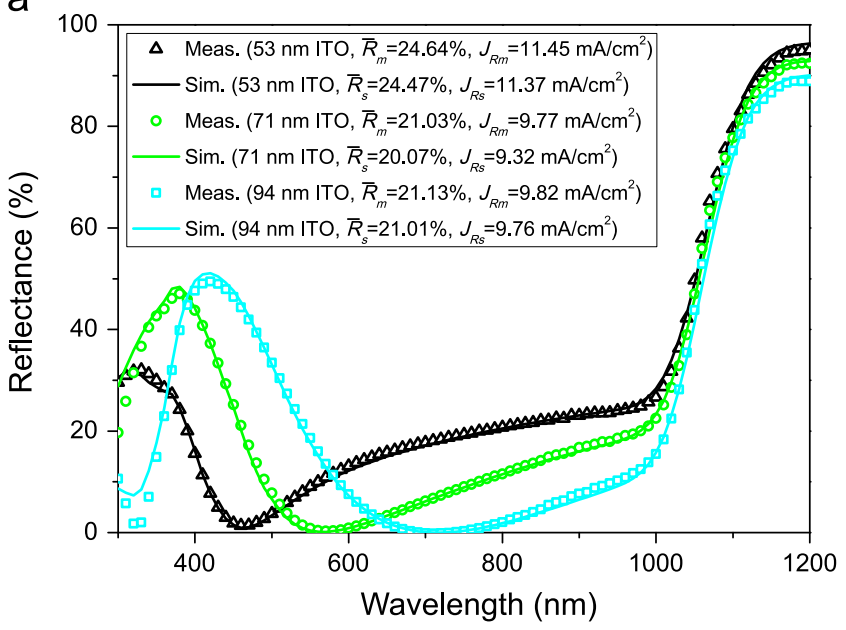

b

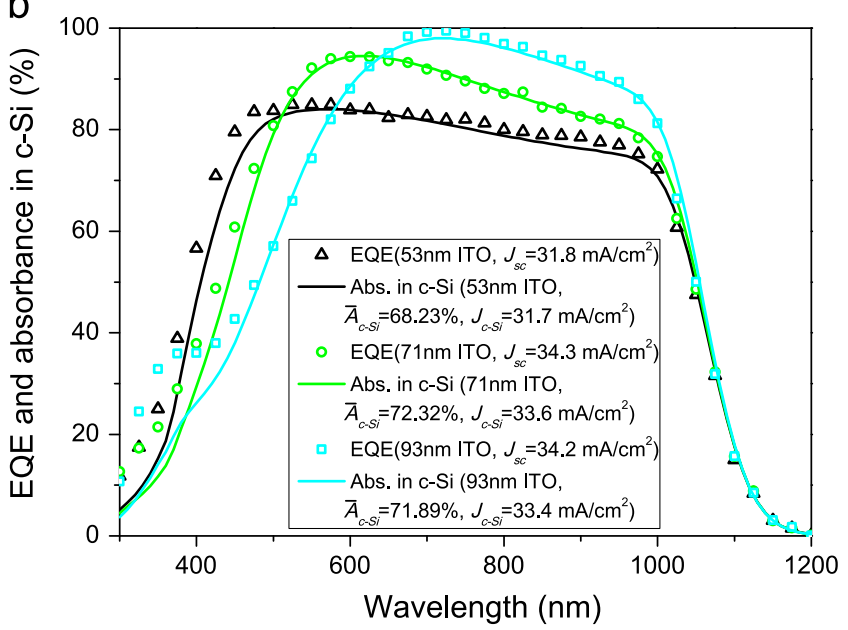

Fig. 6. (a) Reflectance spectra measured on three heterojunction silicon solar cells with different thicknesses of the single-layer AR coating of ITO and the corresponding ASA simulations. (b) EQE measurement and simulated absorbance in c-Si of those heterojunction silicon solar cells.

When the ITO thickness increases, the wavelength at which the reflectance approaches zero is shifted to longer wavelengths. As predicted by the simulations, the $\bar{R}$ calculated from the reflectance measurement is the lowest when the ITO thickness is close to $80 \mathrm{~nm}$. There is excellent agreement between the simulated reflectance (lines) and the measurement (symbols), and $\bar{R}$ calculated from reflectance measurement and simulation are nearly the same. This confirms the accuracy of the optical model and of the optical properties of the layers used as input.

In Fig. 6(b), the measured EQE (symbols) is shown for the three solar cells with different ITO thicknesses and the $J_{\mathrm{sc}}$ is calculated from the EQE spectrum. In the same figure the simulated absorbance in c-Si is presented (lines). The corresponding $\bar{A}_{c-S i}$ and current density calculated from $\bar{A}_{c-S i}$ are given in the legend. This figure confirms the conclusion discussed previously about Fig. 3 that the measured EQE very closely matches the simulated absorbance in c-Si. The minor deviation at shorter wavelengths can be mainly attributed to the current contribution from the a-Si: $\mathrm{H}$ layers. Note that at the wavelength where the reflectance approaches zero (i.e. the device absorbance approaches unity) in the case of $93 \mathrm{~nm}$ thick ITO, the EQE becomes nearly unity. For an ITO thickness of $53 \mathrm{~nm}$ the minimum reflectance occurs at a wavelength of $450 \mathrm{~nm}$. However, at this wavelength the a-Si:H 


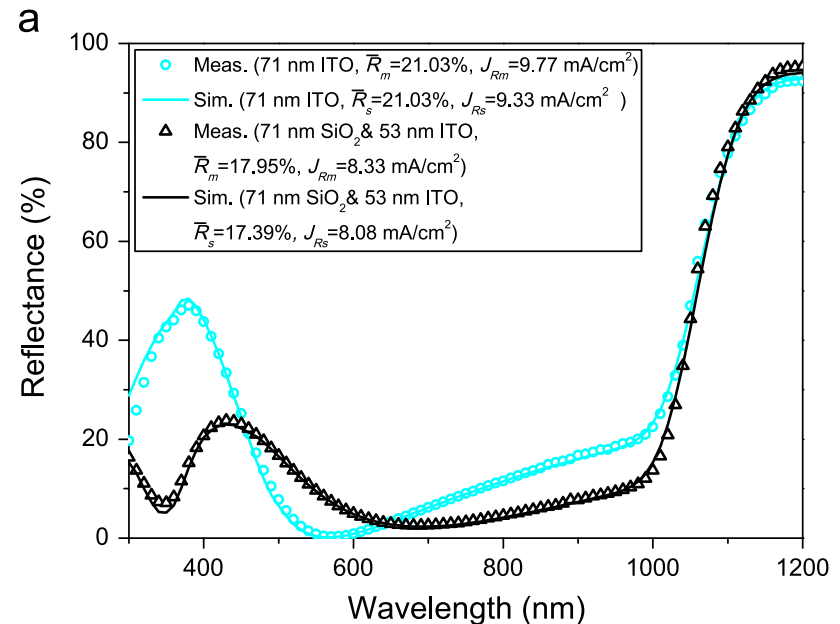

b

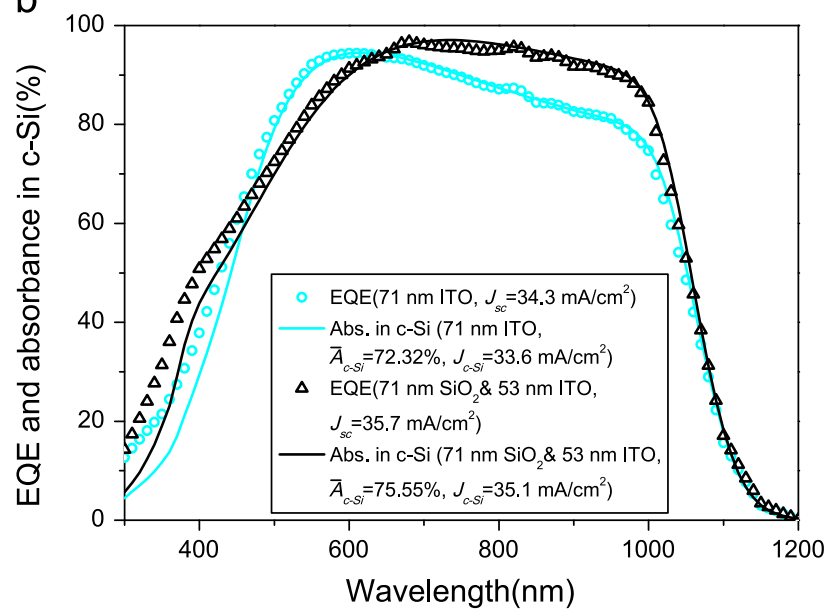

Fig. 7. (a) Reflectance spectra measured on two heterojunction silicon solar cells: one with a single-layer AR coating of $71 \mathrm{~nm}$ ITO and one with a double-layer AR coating of $71 \mathrm{~nm} \mathrm{SiO}{ }_{x}$ and $53 \mathrm{~nm}$ ITO, and the corresponding ASA simulations. (b) EQE measurement and simulated absorbance in c-Si of those heterojunction silicon solar cells.

layers are strongly absorbing. Therefore, the EQE of this cell does not approach unity at any wavelength.

In Fig. 7(a) the reflectances of the optimal devices with a single and a double-layer AR coating are compared. This figure shows that the addition of the $\mathrm{SiO}_{x}$ layer reduces the reflectance (i.e. increases the device absorbance) in the wavelength range of 300 $450 \mathrm{~nm}$ as well as 700-1000 nm. However, the reflectance in the wavelength range of $500-600 \mathrm{~nm}$ is increased. Overall, the $\bar{R}$ is reduced from $21 \%$ to $18 \%$. There is excellent agreement between simulation and the reflectance measurement for the double-layer AR coating as well. By applying a double-layer AR coating, the EQE measurement shows a $J_{\mathrm{sc}}$ increase of $1.4 \mathrm{~mA} / \mathrm{cm}^{2}$ (Fig. 7(b)) which is very close to the increase of $1.5 \mathrm{~mA} / \mathrm{cm}^{2}$ predicted by the simulation of the absorbance in $\mathrm{c}-\mathrm{Si}$. The wavelength range in which the EQE response increases is in line with the range in which the reflection is reduced. In Fig. 8, the $J-V$ characteristics of those two flat heterojunction silicon solar cells with an optimum single-layer and double-layer AR coatings are shown. Due to the $J_{\mathrm{sc}}$ increase of the solar cell with a double-layer AR coating, the efficiency is increased from $17.1 \%$ to $17.6 \%$. Note that the FF drop is not caused by thinner ITO used in the case of the double-layer AR coating since this drop is observed only after $\mathrm{SiO}_{x}$ deposition and $53 \mathrm{~nm}$ thick ITO seems sufficient to maintain the good conductance of the device.

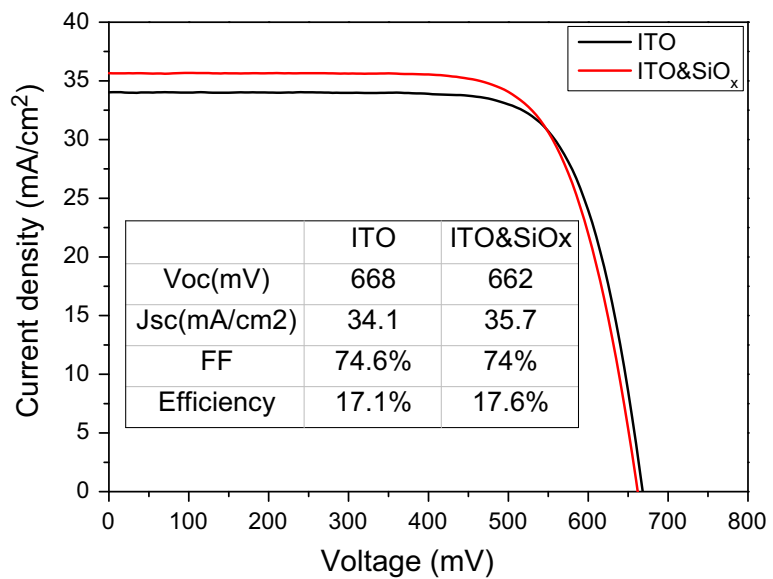

Fig. 8. Measured $J-V$ characteristics of the flat heterojunction silicon solar cells with single-layer and double-layer AR coatings.

\subsection{Optical simulation of the heterojunction silicon solar cell on a textured surface}

Optical simulation has also been carried out for the heterojunction silicon solar cells on textured wafers. It is well known that the reflectance of a textured c-Si wafer is considerably reduced compared to a flat polished wafer, because the fraction of the light initially reflected from the surface can hit the surface for a second time and has another opportunity to enter the wafer. The texture was produced by alkaline etching of c-Si wafers with (100) orientation. The etch exposes the (111) facets, resulting in pyramids with a well-defined steepness of $54.7^{\circ}$. The pyramids are randomly distributed over the surface and have a typical height of $5 \mu \mathrm{m} \mathrm{[15].} \mathrm{A} \mathrm{ray-tracing} \mathrm{model} \mathrm{is} \mathrm{used} \mathrm{to} \mathrm{simulate} \mathrm{the} \mathrm{textured}$ surface. In the model a two-dimensional representation of a single pyramid with a steepness of $54.7^{\circ}$ is used. Periodic boundary conditions are used on either side of the simulation domain to effectively simulate an infinite array of identical pyramids. The optical model uses the matrix formalism described by Macleod [10] to treat incident light traveling obliquely through any multilayer optical coating deposited on the pyramids. Effects due to polarization of light are taken into account. The accuracy of this approach has been previously validated for homojunction c-Si solar cells [14].

In the case where a single-layer AR coating of ITO is used, the $\bar{R}$ and $\bar{A}_{c-S i}$ of the solar cell can be calculated as a function of ITO thickness. The optimum ITO thickness for the solar cell on the textured surface is expected to be lower than that on the flat surface, because light travels through the ITO layer obliquely, increasing the propagation path length of the light in ITO. As shown in Fig. 9(a) the refraction of light when entering the ITO has to be taken into account. The simulation results are shown in Fig. 9 (b). Unlike the flat solar cell, the optimum ITO thickness to obtain the minimum $\bar{R}$ on the textured solar cell, which is $75 \mathrm{~nm}$, is different from that to obtain the maximum $\bar{A}_{c-S i}$, which is $70 \mathrm{~nm}$.

Similarly, when the double-layer AR coating is used on the textured wafer, the optimum combination of ITO and $\mathrm{SiO}_{x}$ thicknesses to obtain the minimum $\bar{R}$ (Fig. 10(a)) is clearly different from that to obtain the maximum $\bar{A}_{c-S i}$ (Fig. 10(b)). Therefore, although a combination of $67 \mathrm{~nm}$ thick ITO and $95 \mathrm{~nm}$ thick $\mathrm{SiO}_{x}$ leads to the minimum $\bar{R}, \bar{A}_{c-S \mathrm{i}}$ is the lowest for a combination of $40 \mathrm{~nm}$ thick ITO and $90 \mathrm{~nm}$ thick $\mathrm{SiO}_{x}$. Since $\bar{A}_{c-\mathrm{Si}}$ dominates $J_{\mathrm{sc}}$, the optimum ITO and $\mathrm{SiO}_{x}$ thicknesses to obtain the maximum $\bar{A}_{c-S i}$ should result in the highest $J_{\text {sc. }}$.

When we compare optical properties of the textured heterojunction silicon solar cell with the optimum single-layer AR 
a

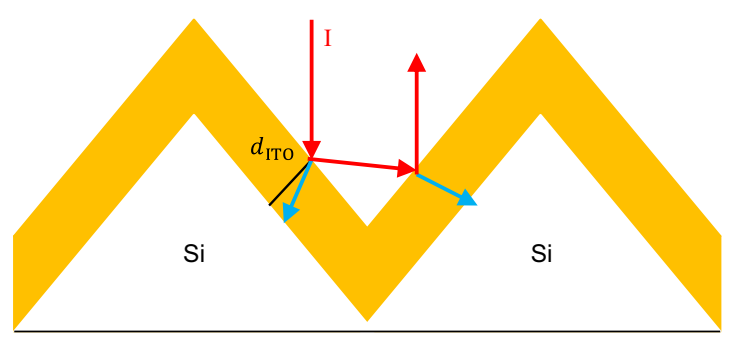

b

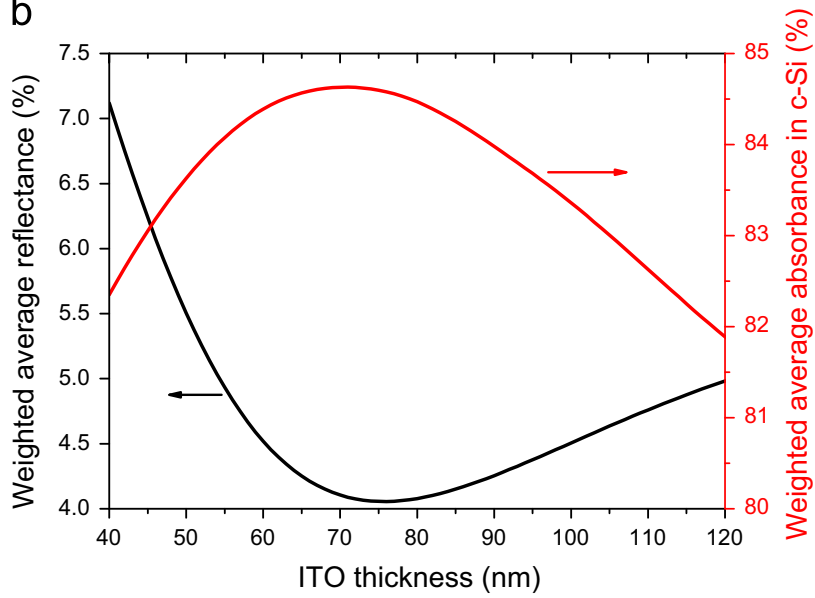

Fig. 9. (a) Light propagation through ITO and (b) the simulated $\bar{R}$ and $\bar{A}_{c-S i}$ as a function of ITO thicknesses.
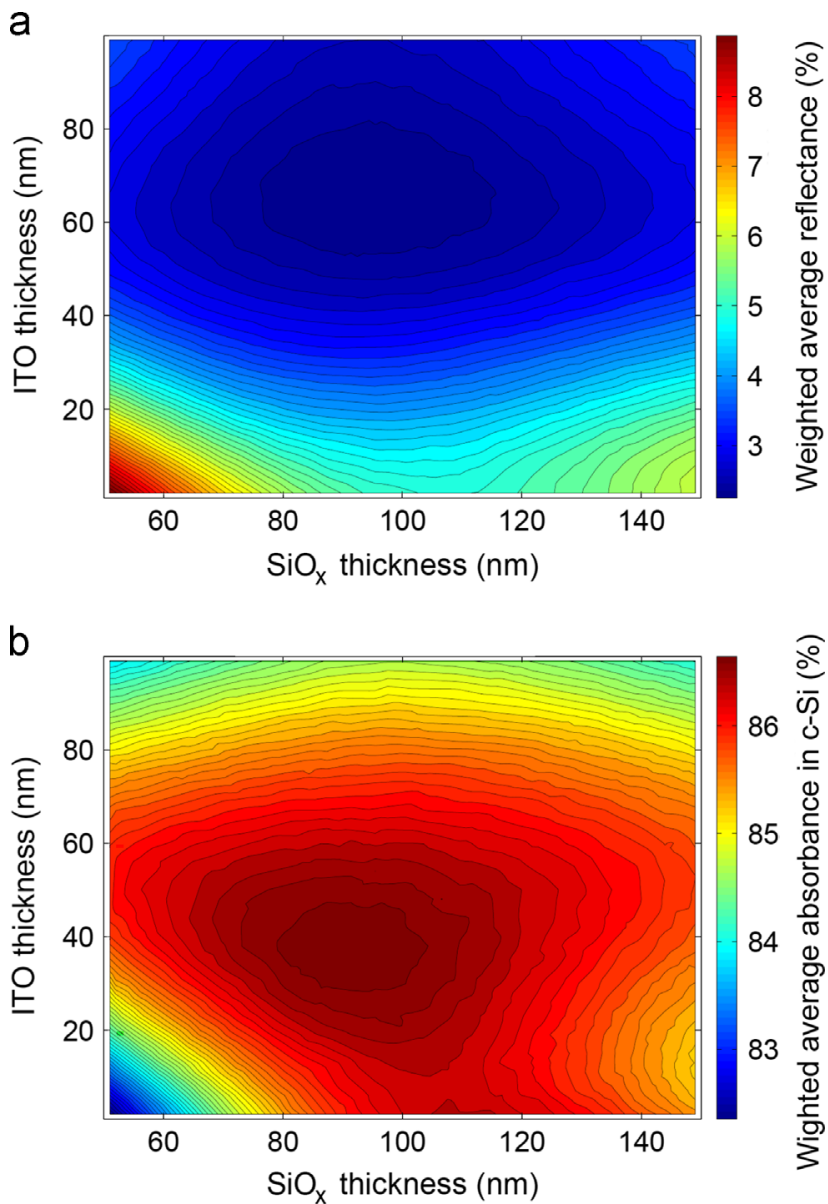

Fig. 10. The simulated (a) $\bar{R}$ and (b) $\bar{A}_{c-\mathrm{Si}}$ as a function of ITO and $\mathrm{SiO}_{x}$ thicknesses. coating of $70 \mathrm{~nm}$ thick ITO shown in Fig. 9(b) to those with the optimum double-layer AR coating shown in Fig. 10(b), the $\bar{R}$ of the device is reduced from $4.1 \%$ to $3.1 \%$ and $\bar{A}_{c-S i}$ increases from $84.6 \%$ to $86.7 \%$. A closer inspection shows that the $2.1 \%$ increase in $\bar{A}_{c-S \mathrm{i}}$ is only partly $(1 \%)$ due to a further reduction of the reflectance. The remaining increase (1.1\%) is due to a reduced parasitic absorption, in particular resulting from a thinner ITO layer. Note that compared to the flat double-layer AR coating, the optimum ITO thickness is now much less ( $40 \mathrm{~nm}$ instead of $60 \mathrm{~nm}$ ) because of the increased importance of reducing parasitic absorption in ITO. To maintain the required total optical AR-coating thickness this ITO thickness reduction is accompanied by an increase in the $\mathrm{SiO}_{x}$ thickness (90 $\mathrm{nm}$ instead of $70 \mathrm{~nm}$ ).

\subsection{Optical measurement of the heterojunction silicon solar cell on a textured surface}

In Fig. 11(a) the reflectances of experimental devices with a single-layer and a double-layer AR coating on textured substrates are compared. Unlike the perfect fitting we obtain for flat cells, there is some deviation in the short wavelength range. We attribute this deviation to sub-wavelength features present on the top of the random array of pyramids that give rise to Rayleigh scattering. This figure shows that the addition of the $\mathrm{SiO}_{x}$ layer reduces the reflectance throughout the wavelength range from $300 \mathrm{~nm}$ to $1100 \mathrm{~nm}$. Overall, the $\bar{R}$ calculated from the reflectance measurements is reduced from $5.62 \%$ in the case of a single-layer
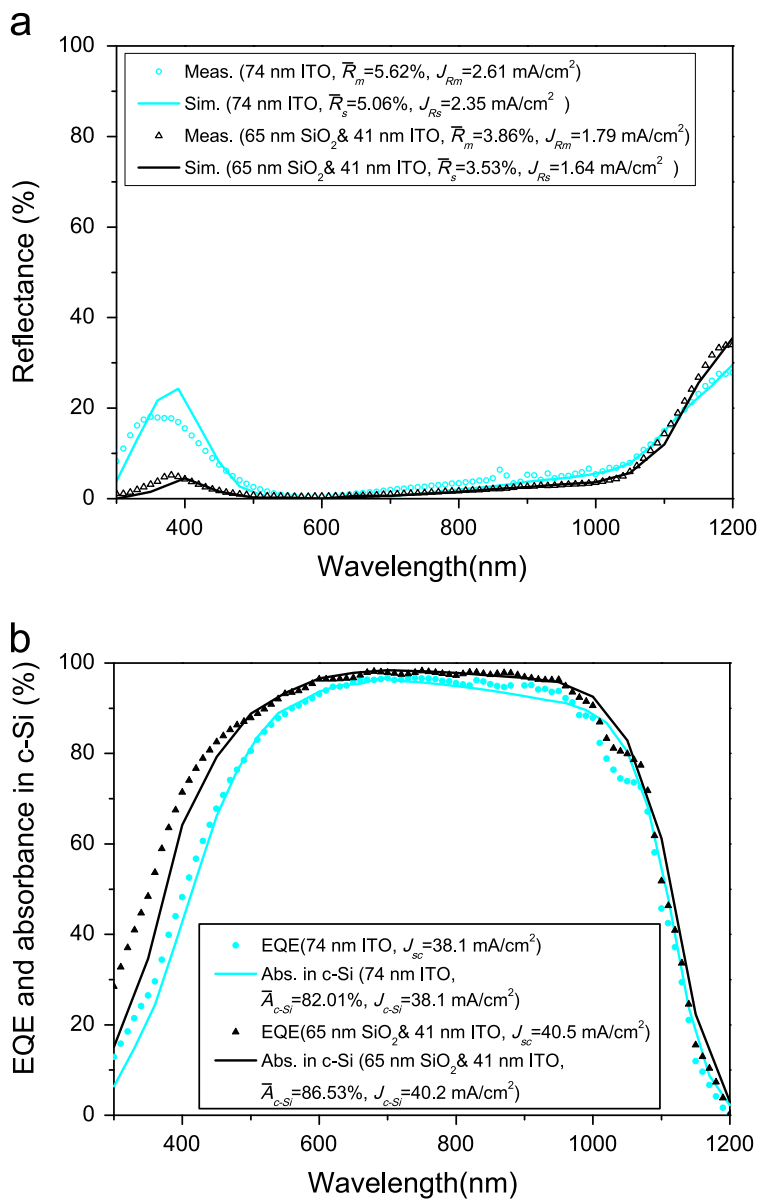

Fig. 11. (a) Reflectance spectra measured on two heterojunction silicon solar cells on textured substrates: one with a single-layer AR coating of $74 \mathrm{~nm}$ ITO and one with a double-layer AR coating of $65 \mathrm{~nm} \mathrm{SiO}_{x}$ and $41 \mathrm{~nm}$ ITO, and the corresponding ASA simulations. (b) EQE measurement and simulated absorbance in c-Si of those heterojunction silicon solar cells. 


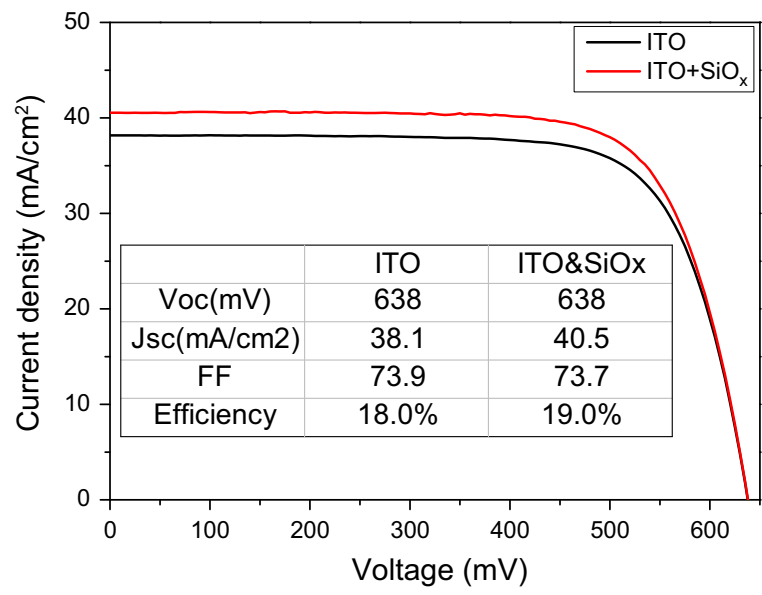

Fig. 12. $J-V$ characteristics of the textured heterojunction silicon solar cell with a single-layer and double-layer AR coating.

AR coating to $3.86 \%$ in the case of a double-layer AR coating, implying a current density increase of $0.82 \mathrm{~mA} / \mathrm{cm}^{2}$. The simulation shows a very similar reduction of $\bar{R}$ from $5.06 \%$ to $3.53 \%$, implying a current density increase of $0.71 \mathrm{~mA} / \mathrm{cm}^{2}$. However, in Fig. 11(b), the EQE measurement shows that the $J_{\mathrm{sc}}$ increases from $38.1 \mathrm{~mA} / \mathrm{cm}^{2}$ to as much as $40.5 \mathrm{~mA} / \mathrm{cm}^{2}$ by applying a doublelayer AR coating. The $J_{\mathrm{sc}}$ increase of $2.4 \mathrm{~mA} / \mathrm{cm}^{2}$ is clearly larger than what can be explained from reflection reduction alone. However, the simulated $\bar{A}_{c-S i}$ takes into account the reduced parasitic absorption in ITO and shows a current density increase of $2.1 \mathrm{~mA} / \mathrm{cm}^{2}$ that is very close to the experimentally obtained $J_{\mathrm{sc}}$ increase. This again proves that the effectiveness of anti-reflection can be better demonstrated by the absorbance in c-Si of the heterojunction silicon solar cell. Note that there is a mismatch between EQE and the absorbance in c-Si at wavelengths from $1000 \mathrm{~nm}$ to $1100 \mathrm{~nm}$, which is not observed in the flat cells. This difference is probably due to the fact that the passivation of the textured wafer surface is not good enough so that recombination losses become more obvious for carriers generated by longwavelength light. That is, a large fraction of those carriers generated far away from the depletion region have an increased probability of recombination. Lower $V_{\text {oc }}$ generally measured in the textured solar cells than in the flat cells also supports this explanation.

The drop of the $V_{\text {oc }}$ on the textured surface compared to the flat surface (Fig. 8) is mainly due to our insufficient wafer cleaning of textured wafers. The contaminants left from the texturing process cannot be effectively removed, leading to an increased surface recombination velocity on the textured surface compared to the flat surface. Eventually on the textured wafer, using a double-layer anti-reflective coating, the efficiency of the heterojunction silicon solar cell is increased from $18.0 \%$ to $19.0 \%$ as shown in Fig. 12.

\section{Conclusion}

In this contribution, we present optical simulations for flat and textured heterojunction silicon solar cells with our ASA software. Our simulations show excellent agreement with reflectance measurements on real devices, indicating the accuracy of the model used. We have shown that optical optimization of textured heterojunction solar cells needs to be carried out especially in relation to the $\bar{A}_{c-S \mathrm{i}}$ and not in relation to the $\bar{R}$ since parasitic absorption plays an important role. Based on this approach we have designed a double-layer AR coating of $\mathrm{SiO}_{x}$ and ITO for the heterojunction silicon solar cell. Comparing to the single-layer AR coating of only ITO, the double-layer AR coating not only shows a pronounced reduction of the reflectance, but also decreases the optically required ITO thickness thereby reducing parasitic absorption and increasing $\bar{A}_{c-S i}$.

The heterojunction silicon solar cell that we fabricated on the flat surface shows an increase of $J_{\mathrm{sc}}$ from $34.3 \mathrm{~mA} / \mathrm{cm}^{2}$ to $35.7 \mathrm{~mA} / \mathrm{cm}^{2}$ in agreement with our optical simulation. The $J_{\text {sc }}$ increase leads to the increase of the solar-cell efficiency from $17.1 \%$ to $17.6 \%$. For devices on textured surfaces the $J_{\mathrm{sc}}$ increases from $38.1 \mathrm{~mA} / \mathrm{cm}^{2}$ to $40.5 \mathrm{~mA} / \mathrm{cm}^{2}$. This $J_{\mathrm{sc}}$ increase leads to the increase of the solar-cell efficiency from $18.0 \%$ to $19.0 \%$.

\section{Acknowledgments}

The work is funded by Energy Research Centre of The Netherlands. We would like to thank M. Tijssen and S. Heirman for their technical support. We also would like to thank H. Tan, J. Meerwijk, D. Deligiannis and other colleagues for their helpful discussion.

\section{References}

[1] T. Kinoshita, D. Fujishima, A. Yano, A. Ogane, S. Tohoda, K. Matsuyama, Y. Nakamura, N. Tokuoka, H. Kanno, H. Sakata, M. Taguchi, E. Maruyama, The approaches for high efficiency HIT solar cell with very thin $(<100 \mu \mathrm{m})$ silicon wafer over 23\%, in: Proceedings of the 26th European Photovoltaic Solar Energy Conference, 2011, pp. 871-874.

[2] A. Descoeudres, Z.C. Holman, L. Barraud, S. Morel, S. De Wolf, C. Ballif, $>21 \%$ efficient silicon heterojunction solar cells on n- and p-type wafers compared, IEEE Journal of Photovoltaics 3 (1) (2013) 83-89.

[3] S. De Wolf, A. Descoeudres, Z.C. Holman, C. Ballif, High-efficiency silicon heterojunction solar cells: a review, Green 2 (2012) 7-24.

[4] E. Maruyama, A. Terakawa, M. Taguchi, Y. Yoshimine, D. Ide, T. Baba, M. Shima, H. Sakata, M. Tanaka, Sanyo's challenges to the development of high-efficiency HIT solar cells and the expansion of HIT business, in: Proceedings of the 4th IEEE World Conference on Photovoltaic Energy Conversion, vol. 2, 2006, p. 1455-1460.

[5] Z.C. Holman, A. Descoeudres, L. Barraud, F.Z. Fernandez, J.P. Seif, S. De Wolf C. Ballif, Current losses at the front of silicon heterojunction solar cells, IEEE Journal of Photovoltaics 2 (2012) 7-15.

[6] M. Zeman, J. Krc, Optical and electrical modeling of thin-film silicon solar cells, Journal of Materials Research 23 (2008) 889-898.

[7] B.E. Pieters, J. Krc, M. Zeman, Advanced numerical simulation tool for solar cells-ASA5, in: Proceedings of the 4th IEEE World Conference on Photovoltaic Energy Conversion, 2006, p. 1513.

[8] W. Theiss, Scout Thin Film Analysis Software Handbook, W.Theiss Hard- and Software for Optical Spectroscopy, 2004

[9] E. Palik, Handbook of Optical Constants of Solids, Academic Press, San Diego 1998.

[10] H.A. Macleod, Thin-film Optical Filters, fourth ed., Taylor \& Francis Group, Boca Raton, 2010.

[11] D. Thorp, S.R. Wenham, Ray-tracing of arbitrary surface textures for lighttrapping in thin silicon solar cells, Solar Energy Materials and Solar Cells 48 (1997) 295-308

[12] T. Yagi, Y. Uraoka, T. Fuyuki, Ray-trace simulation of light trapping in silicon solar cell with texture structures, Solar Energy Materials and Solar Cells 90 (2006) 2647-2656.

[13] Z.C. Holman, M. FilipiC, A. Descoeudres, S. De Wolf, F. Smole, M. Topič, C. Ballif, Infrared light management in high-efficiency silicon heterojunction and rearpassivated solar cells, Journal of Applied Physics 113 (2013) 013107.

[14] R. Santbergen, R.J.C. van Zolingen, The absorption factor of crystalline silicon PV cells: a numerical and experimental study, Solar Energy Materials and Solar Cells 92 (2008) 432-444.

[15] J.D. Hylton, A.R. Burgers, W.C. Sinke, Alkaline etching for reflectance reduction in multicrystalline silicon solar cells, Journal of the Electrochemical Society 151 (2004) G408-G427. 